\title{
Formation of powder materials under the combined influence of pulse laser radiation and electric arc discharge
}

\author{
Dmitriy Kochuev* \\ Vladimir State University, Vladimir 600000, Russia
}

\begin{abstract}
In this paper presents an assessment of the efficiency of the synthesis of nanoscale spherical powder materials by the combined action of ultrashort laser pulses and electric arc discharge. Experimentally determined optimal from the point of view of technological effectiveness and efficiency, the conditions conducive to the spread of eclectic arc discharge by laser-induced plasma channel. The scheme of experimental work, the results achieved.
\end{abstract}

For laser ablation treatment, the use of the combined approach is due to the significantly lower cost of the electric arc discharge energy compared to the laser one, such approaches are presented in [1-2]. It is known that when processing metals by laser radiation, effective ablation occurs under the condition of heat accumulation. When processing with ultrashort laser pulses, thermal energy is accumulated as a result of relaxation processes both in the structure of the processed material and as a result of the action of a laser-induced plasma torch. Such actions can increase the energy supplied to the treatment area and at the same time preserve the locality of the action limited by the region of propagation of laser-induced plasma. The lifetime of laser-induced plasma and relaxation of thermal processes significantly exceed the duration of the laser pulse. Providing a tangible positive effect on the dynamics of laser ablation pumping laser-induced channel arc discharge energy will significantly increase the energy supplied to the processing area and increase its lifetime Using this method of treatment, the laser energy is used to form a conducting plasma channel used to transport the electric arc discharge to the surface of the sample [3]. The use of a laser beam as a means of forming a conductive region in space allows not only to exclude the formation of impurities in the resulting powder material, but also to carry out a dynamic change in the position of the processing area on the surface of the sample. Using this feature, it is possible to scan with a laser beam as a fraction of the extended electrode, which will avoid bulk melting of the sample and maintain a high locality of energy application.

Figure 1 shows the scheme of experimental setup. The paper presents the surface treatment of titanium (11) by the combined action of ultrashort laser pulses and electric arc discharge (6) [4]. The optimal focal length of the optical system (2) was determined to form a region of laser-induced plasma in the caustic region of the required length.

\footnotetext{
*Corresponding author:_b_@mail.ru
} 


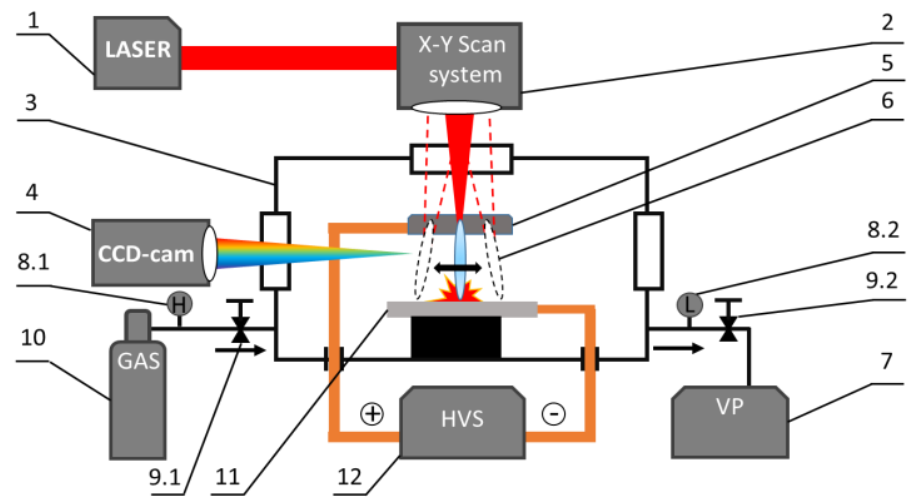

Fig. 1. Scheme of the combined treatment process.

Laser beam scanning was performed along an extended electrode (5). Thus, the distance from the laser-induced plasma channel to the electrode remained unchanged, the high ablation dynamics of the material remained, the bulk melting of the sample was not observed. The laser-induced plasma torch was initiated by a laser radiation source "TETA-10" (1) with a pulse duration of $280 \mathrm{FS}$, pulse energy of $150 \mu \mathrm{j}$, with a wavelength of $1030 \mathrm{~nm}$. The focus area was selected in such a way that the ablation products did not act as a framework for the propagation of an electric arc discharge like the propagation of an electric arc on dust particles described in [5]. The concentration of free electrons in the laser-induced plasma channel is an important parameter. Increasing the concentration of free electrons in the volume of the laser-induced plasma channel was carried out by increasing the pressure in the working chamber (3). The concentration of free electrons was estimated by analyzing the spectra of the laser-induced plasma channel using the approaches described in [6]. Highpurity argon from the cylinder (10) was used as a gas in the working chamber, the working pressure was maintained in the range of 8-10 bar. The volume of the chamber was pumped out before filling with a vacuum pump (7). High pressure monitoring was carried out by the sensor (8.1), regulated by the valve (9.1). The discharge pressure was controlled by a sensor (8.2) and regulated by a valve (9.2). The high-voltage pulse was formed by the generator (12), the voltage varied in the range from 20 to $30 \mathrm{kV}$, the energy in the pulse of the electric arc was $0.05-0.1 \mathrm{j}$. the transition of the arc was carried out from the positive electrode (5) to the negative electrode-sample (11). The process was recorded by CCD camera (4).

\section{References}

1. Lyubimov V.V., Kozyr D.V., Gnidina I.V. Izvestiya TulGU. Tech. science.10 68 (2015)

2. Kozyr D.V. Izvestiya TulGU. Tech. science.11 141 (2013)

3. Ivashchenko A.V., Kochuev D.A, Chkalov R.V. et al. J. Phys. Conf. Ser. 1164012021 (2019)

4. Ivashchenko A., Kochuev D., Chkalov R. Mater. Today Proc. 11465 (2019)

5. Kukushkin A.B., Rantsev-Kartinov V.A. Microsystem technology. 322 (2002)

6. Kochuev D., Chkalov R., Chernikov A. Mater. Today Proc. (2019) (in press). 